\title{
担载型中空纤维混合导体氧渗透膜的制备与性能研究
}

\author{
刘郑㾦，朱佳伟，金万勤 \\ (南京工业大学 化学化工学院, 材料化学工程国家重点实验室, 南京 210009)
}

摘 要:采用干湿法纺丝技术制备 $\mathrm{Sr}_{0.7} \mathrm{Ba}_{0.3} \mathrm{Fe}_{0.9} \mathrm{Mo}_{0.1} \mathrm{O}_{3-\delta}(\mathrm{SBFM})$ 中空纤维支撑体，以 $\mathrm{Nb}_{2} \mathrm{O}_{5}$ 掺杂的 $\mathrm{SrCo}_{0.8} \mathrm{Fe}_{0.2} \mathrm{O}_{3-\delta}$

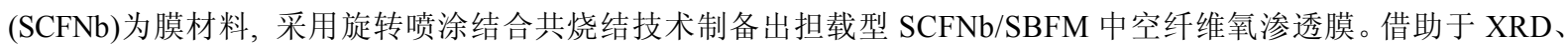
SEM、热膨胀分析、透氧及膜反应性能测试等手段, 分别对样品的晶相结构、膜微观结构、支撑体与膜层的烧 结行为、膜的氧渗透通量及膜反应性能进行了研究。结果表明, 膜层与支撑体的晶相结构仍保持钻钠矿主体相。 支撑体具有单一海绵孔/指状孔结构, 膜厚为 $5 \mu \mathrm{m}$ 且致密无缺陷, 膜层与支撑体结合良好。在 $900^{\circ} \mathrm{C}$ 时, 氧渗透通 量达到 $0.74 \mathrm{~mL} /\left(\mathrm{cm}^{2} \cdot \mathrm{min}\right) 。 850^{\circ} \mathrm{C}$ 下甲烷部分氧化膜反应稳定操作超过 $200 \mathrm{~h}$, 稳态下氧渗透通量为 $4.5 \mathrm{~mL} /\left(\mathrm{cm}^{2} \cdot \mathrm{min}\right)$ 。 研究表明, 担载型 SCFNb/SBFM 中空纤维氧渗透膜具有较高的氧渗透通量, 同时具有良好的膜反应稳定性。

关 键 词: 担载型中空纤维膜; 混合导体膜; 透氧; 膜反应

中图分类号: TQ174 文献标识码: A

\section{Preparation and Characterization of Mixed-conducting Supported Hollow Fiber Membrane}

\author{
LIU Zheng-Kun, ZHU Jia-Wei, JIN Wan-Qin
}

(State Key Laboratory of Materials-Oriented Chemical Engineering, College of Chemistry and Chemical Engineering, Nanjing Tech University, Nanjing 210009, China)

\begin{abstract}
Sr}_{0.7} \mathrm{Ba}_{0.3} \mathrm{Fe}_{0.9} \mathrm{Mo}_{0.1} \mathrm{O}_{3-\delta}$ (SBFM) hollow fiber supports were prepared by dry-wet spinning technology. $\mathrm{SrCo}_{0.8} \mathrm{Fe}_{0.2} \mathrm{O}_{3-\delta}$ doped $\mathrm{Nb}_{2} \mathrm{O}_{5}$ ( $\mathrm{SCFNb}$ ) was selected as membrane material, $\mathrm{SCFNb} / \mathrm{SBFM}$ supported hollow fiber membranes were successfully prepared by a combined spin-spraying and co-sintering method. XRD, SEM, thermal expansion technique, oxygen permeability and membrane reaction tests were utilized to characterize the crystal phase structures, microstructure, sintering behavior, oxygen permeation flux, and reaction performance of membranes and/or supports, respectively. XRD patterns show that the membranes and supports are composed of perovskite main phase. SEM images indicate that the support has an asymmetric structure with a sponge-like microporous/finger-like porous structure. The membrane surface is dense and crack-free, and the membrane layer with a thickness of about $5 \mu \mathrm{m}$ is well bonded with the support. At $900^{\circ} \mathrm{C}$, the oxygen permeation flux of the membrane is $0.74 \mathrm{~mL} /\left(\mathrm{cm}^{2} \cdot \mathrm{min}\right)$. No degradation of performance is observed in the membrane reactor under partial oxidation of methane during continuously operating for $200 \mathrm{~h}$ at $850^{\circ} \mathrm{C}$, and the oxygen permeation flux can reach $4.5 \mathrm{~mL} /\left(\mathrm{cm}^{2} \cdot \mathrm{min}\right)$. It is demonstrated that the SCFNb/SBFM supported hollow fiber membrane exhibits high oxygen permeation flux and good stability of the membrane reactor.
\end{abstract}

收稿日期：2014-12-16; 收到修改稿日期：2015-02-02

基金项目: 国家 973 计划项目(2009CB623406); 教育部创新团队发展计划(IRT13070); 江苏高校优势学科建设工程基金(PAPD) National Basic Research Program of China (2009CB623406); Innovative Research Team Program by the Ministry of Education of China (IRT13070); Project of Priority Academic Program Development of Jiangsu Higher Education Institutions (PAPD)

作者简介: 刘郑斿(1984-), 男, 博士研究生. E-mail: flyice-lzk@163.com

通讯作者: 金万勤，教授. E-mail: wqjin@njtech.edu.cn 
Key words: supported hollow fiber; mixed-conducting membrane; oxygen permeation; membrane reaction

混合氧离子电子导体致密透氧膜是一种具有 氧离子及电子导电性能的陶瓷膜, 其氧传输过程 中不需要外加电路, 且在高温下对氧具有绝对的 选择性 ${ }^{[1]}$ 。这些优异的特性使得此类材料在空气分 离、固体燃料电池、膜反应器和富氧燃烧等领域具 有广阔的应用前景。

在实际应用中，混合导体透氧膜不仅需要氧 通量高, 而且还要化学稳定。降低膜厚是提高氧 通量的一种可行方法, 如开发非对称膜(担载膜), 即在多孔支撑体上采用各种手段沉积一层薄的致 密膜 ${ }^{[2]}$ 。相比于片式膜，管式膜具有易于工业放大 和高温密封简单的优点。Ito 等 ${ }^{[3]}$ 采用浆料涂覆法 在多孔管式支撑体上制备了膜厚为 $50 \mu \mathrm{m}$ 的 $\mathrm{SrCo}_{0.9} \mathrm{Nb}_{0.1} \mathrm{O}_{3-\text { - }}$ 致密膜。Kawahara 等 ${ }^{[4]}$ 采用滑涂法 在多通道结构的支撑体上制备了膜厚为 $60 \mu \mathrm{m}$ 的 $\mathrm{La}_{0.6} \mathrm{Sr}_{0.4} \mathrm{Ti}_{0.3} \mathrm{Fe}_{0.7} \mathrm{O}_{3}$ 薄膜。Fan 等 ${ }^{[5]}$ 采用流延镀膜 法制备了 $\mathrm{SrCo}_{0.8} \mathrm{Fe}_{0.2} \mathrm{O}_{3-\delta}$ 管式非对称膜。近年来, 各国的科学家通过相转化联合烧结技术制备的另 一种管式非对称膜, 即中空纤维膜, 该膜结构具 有管径小、管壁薄的特点。英国的 $\mathrm{Li}^{[6-8]}$ 、德国的 $\operatorname{Caro}^{[9]}$ 、国内的王海辉 ${ }^{[10-11]}$ 和谭小耀 ${ }^{[12-14]}$ 等课题 组专注于中空纤维透氧膜的研究, 将其用于氧气 分离, 获得了比传统的片式和管式膜高出许多的 氧渗透通量。

中空纤维透氧膜用于甲烷部分氧化膜反应中, 有两个因素会导致膜反应性能的降低 ${ }^{[15]}$ : 一是膜 表面直接接触还原性气氛致使膜表面被腐蚀; 二 是膜表面与催化剂之间发生固相反应。基于此，本 课题组提出了一种多孔一致密结构的双层复合膜反 应器 ${ }^{[16]}$ 。双层复合膜是由不同材料的多孔层和致密 层紧密结合而成：由耐还原性材料组成的多孔层 减少反应气氛对致密层主体的腐蚀，而由高氧渗 透性能材料组成的致密层用于有效地传递氧离子, 从而使膜反应稳定性有所提高。

本工作以课题组前期开发具有良好耐还原性 的 SBFM 和高氧渗透性能的 SCFNb 分别作为支撑 体和膜层材料，采用旋转喷涂法制备担载型 $\mathrm{SCFNb} / \mathrm{SBFM}$ 中空纤维氧渗透膜, 并对其微观结 构、烧结行为、氧渗透性能和甲烷部分氧化膜反 应性能进行系统地考察和研究。

\section{1 实验方法}

\section{1 粉体和担载型中空纤维膜的制备}

BSFM 和 SCFNb 粉体采用传统的固相反应法进 行合成，合成方法详见文献[17]。采用干湿法纺丝技 术制备 SBFM 中空纤维支撑体生坏，具体过程如下： 将 SBFM 粉体与分散剂混合并置于球磨机内以有机 溶剂作为介质连续球磨 $4 \mathrm{~h}$, 之后加入聚醚砜再连 续球磨 $24 \mathrm{~h}$ 得到分散均匀的纺丝液。纺丝液经抽真 空脱泡处理后倒入注射器中, 再通过注射泵推入纺 丝头后经过一定的干纺过程进入外凝固浴槽(干纺 距离 $25 \mathrm{~cm}$, 纺丝液流速为 $6 \mathrm{~mL} / \mathrm{min}$, 芯液流速为 $18 \mathrm{~mL} / \mathrm{min}$ ), 干燥后得到 SBFM 中空纤维支撑体生 坏。将 SCFNb 粉体通过球磨分散在乙醇溶液中, 配 制成 SCFNb 悬浮溶液用于喷涂。采用旋转喷涂法在 中空纤维支撑体生坏上制备一层薄的 SCFNb 涂层, 具体方法见文献[18]。制备好的担载型 $\mathrm{SCFNb} /$ $\mathrm{SBFM}$ 中空纤维氧渗透膜生坏在 $1160^{\circ} \mathrm{C}$ 下焙烧 $2 \mathrm{~h}$ 。

\section{2 样品的表征}

采用德国 Bruker 公司的 D8-Advance X 射线粉 末衍射仪对样品的晶相结构进行分析。通过扫描电 镜(SEM, QUANTA-2000)分析膜管的微观结构和形 貌。膜层与中空纤维支撑体的界面采用 HORIBAEMAX 能量弥散 $X$ 射线探测器(EDX)进行元素分 析。采用热膨胀仪(Netzsch, DIL 402C)分析膜层与支 撑体的烧结行为。

\section{3 氧渗透性能及膜反应性能的测定}

膜的氧渗透性能、甲烷部分氧化反应性能及稳 定性考察在自建的高温膜反应器装置进行 ${ }^{[19]}$ 。膜管 由两根致密氧化铝管固定，采用银胶进行密封，然 后装入膜组件内(如图 1 所示)。将组件置于电炉恒 温区间，以 $2^{\circ} \mathrm{C} / \mathrm{min}$ 速度升温至 $930^{\circ} \mathrm{C}$, 保温 $2 \mathrm{~h}$ 后将电炉温度以 $2^{\circ} \mathrm{C} / \mathrm{min}$ 速度调至测试温度, 通入 气体稳定 $0.5 \mathrm{~h}$ 以上, 开始透氧性能(或甲烷部分 氧化反应性能)的测定。甲烷部分氧化测定时, $\mathrm{Ni} / \mathrm{Al}_{2} \mathrm{O}_{3}$ 催化剂预先填充在管内。氦气(或氦气 + 甲 烷)和空气分别引入膜的内侧和外侧。渗透侧的氧气 (或反应产物)经氦气吹扫进入气相色谱仪(Shimadzu GC-8A)进行在线分析。膜的密封效果通过检测膜组 件渗透侧气体中的氮气浓度进行判断。 


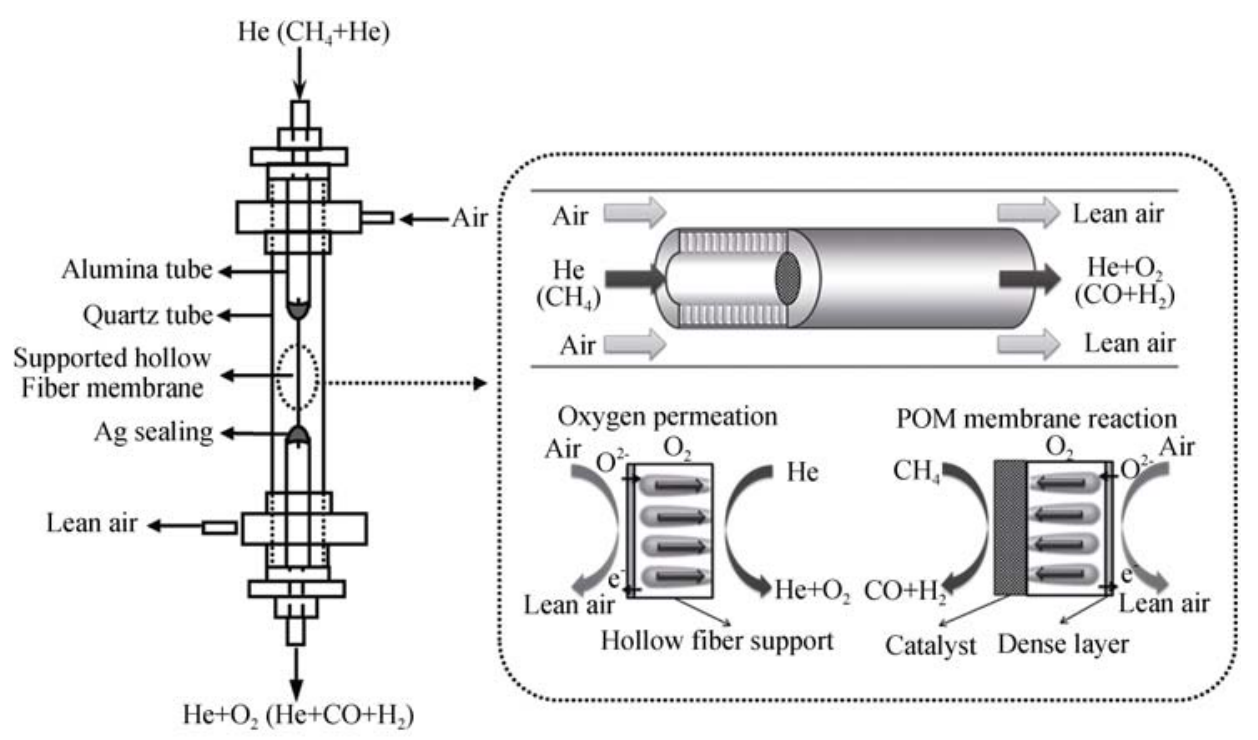

图 1 膜组件及膜反应器示意图

Fig. 1 Schematic diagram of membrane module and membrane reactor

\section{2 结果与讨论}

\section{1 样品的晶体结构分析}

图 2 为 SBFM 中空纤维支撑体和 $\mathrm{SCFNb}$ 膜层 的 XRD 图谱, 由图可知, 经过 $1160^{\circ} \mathrm{C}$ 烧结 $2 \mathrm{~h}$ 后的 $\mathrm{SCFNb}$ 膜层和 SBFM 中空纤维支撑体的晶相基本相 同, 都含有立方结构的钙钛矿主相, 但是 SBFM 支 撑体在 $2 \theta \approx 30^{\circ}$ 处出现了一个很小的杂峰, 对应 $\mathrm{BaFeO}_{2.8}(\mathrm{PDF}$ 54-0966)。这可能是由于固相反应过 程中形成的 $\mathrm{BaFeO}_{2.8}$ 中间相并未完全与其他中间相 固溶形成均相固溶体 $\mathrm{Sr}_{0.7} \mathrm{Ba}_{0.3} \mathrm{Fe}_{0.9} \mathrm{Mo}_{0.1} \mathrm{O}_{3-\delta}$, 也可 能是在烧结过程中晶界及外表面的钡离子和铁离子 的富集形成 $\mathrm{BaFeO}_{2.8}$ 相所致 ${ }^{[1]}$ 。这个非纯相 $\mathrm{BaFeO}_{2.8}$ 在耐还原性气氛下也具有一定氧渗透性能, 且含量 少, 对膜的氧渗透及膜反应影响不大。

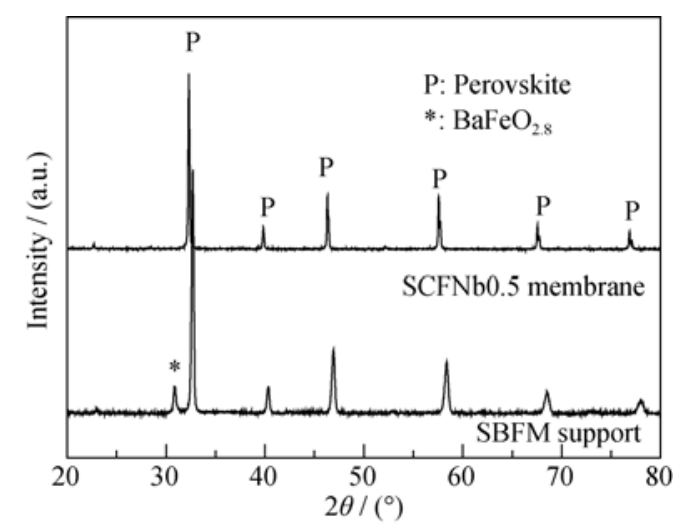

图 $2 \mathrm{SCFNb}$ 膜层和 $\mathrm{SBFM}$ 支撑体的 XRD 图谱

Fig. 2 XRD patterns of SCFNb membrane and SBFM support

\section{2 膜与支撑体的烧结行为分析}

采用本课题组前期提出的“协同收缩”思想 ${ }^{[2]}$ 制 备致密无缺陷的担载膜, 关键在于保证支撑体和膜 层的烧结性能的匹配。样品的烧结性能包括温度升 高过程中的收缩速率和最终的烧结程度。通过热膨 胀仪在线测定样品长度随着时间和温度的变化关系， 可以同时得到样品的烧结收缩曲线和烧结速率曲 线。图 3 为中空纤维支撑体和膜层在空气气氛下的 烧结性能曲线。如图 3(a)所示, 在测试温度范围内膜 层与支撑体的烧结收缩过程不一致。在 $200 \sim 400^{\circ} \mathrm{C}$ 之 间膜层和支撑体都有轻微收缩，分别由膜层中溶剂 和分散剂及支撑体中聚醚砜的烧除所造成。在烧结 过程中，膜层和支撑体都在 $700^{\circ} \mathrm{C}$ 开始烧结，在 $1200^{\circ} \mathrm{C}$ 时膜层和支撑体的线性收缩率分别为 $30.4 \%$ 和 $50.9 \%$ 。为了更加清晰观察样品在烧结过程中长度 的变化, 需要结合烧结收缩速率随温度变化 $(\mathrm{d} L / \mathrm{d} t \sim T)$ 的曲线进行分析。图 3(b)给出了膜层和 支撑体烧结收缩速率曲线, 从图中可以看出, 在烧 结过程中 $\left(>700^{\circ} \mathrm{C}\right)$, 支撑体的烧结速率随着温度的 升高明显增大。相比之下, 膜层的烧结速率在 $810^{\circ} \mathrm{C}$ 和 $1000^{\circ} \mathrm{C}$ 达到最大，高于 $1100^{\circ} \mathrm{C}$ 后开始减小。当烧 结温度高于 $1000^{\circ} \mathrm{C}$ 时, 支撑体的烧结速率大于膜层 的烧结速率，因此，这个烧结阶段膜层的收缩限制 了支撑体的收缩, 从而导致裂纹和缺陷的出现。通常 情况下, 材料的线性收缩受到多个因素的影响 ${ }^{[20]}$, 例 如陶瓷粉料粒径分布、陶瓷粉料与粘结剂的比例和 烧结条件等。在本实验中, 为了避免膜在烧结过程中 出现缺陷, 采用了慢速加热 $\left(0.5 \sim 1.5{ }^{\circ} \mathrm{C} / \mathrm{min}\right)$ 。 

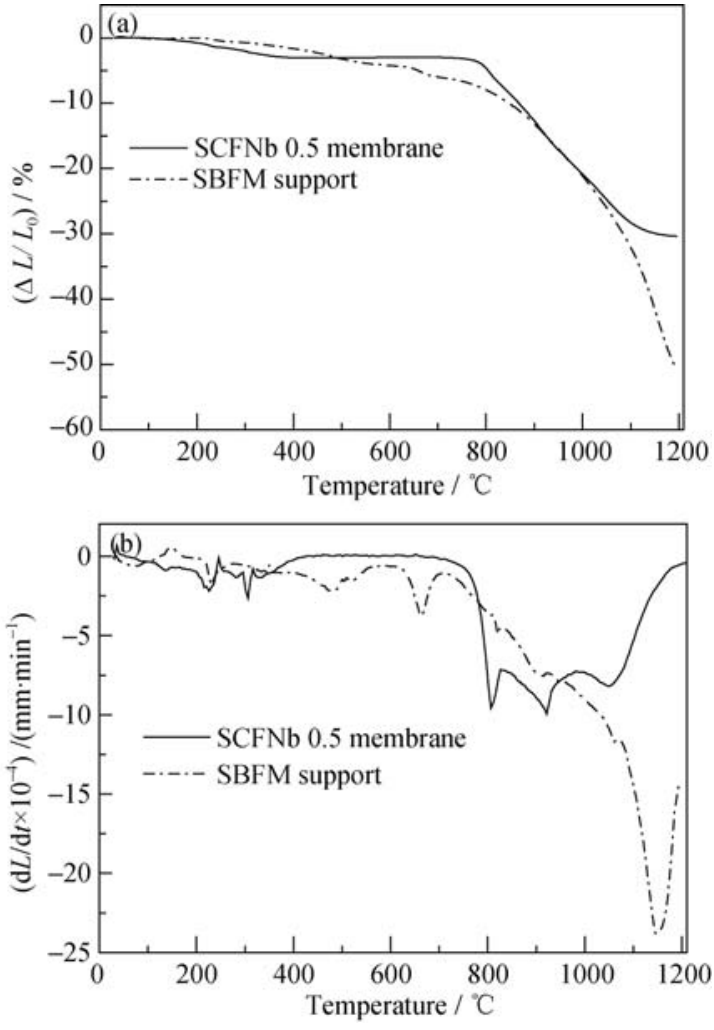

图 3 膜层与支撑体的烧结收缩曲线和烧结速率曲线

Fig. 3 Shrinkage curves (a) and shrinkage rate curves (b) of $\mathrm{SCFNb}$ membrane layer and SBFM support layer

\section{3 担载型中空纤维膜的微观形貌分析}

图 4 为经过 $1160^{\circ} \mathrm{C}$ 烧结 $2 \mathrm{~h}$ 后的担载型 $\mathrm{SCFNb} /$ SBFM中空纤维氧渗透膜及相同高温处理的SBFM中 空纤维支撑体表面的微观形貌。从膜的断面SEM照 片(图4(a)、(b))可以看出, 所制备的氧渗透膜具有非 对称结构, 支撑体具有单一海绵孔/指状孔结构, 指 状大孔在内侧, 海绵状微孔在外侧, 在支撑体外表 面一层致密膜层, 膜层厚度约为 $5 \mu \mathrm{m}$, 且膜层与支 撑体界面结合良好。为了进一步确定膜层与支撑体 界面结合情况, 对界面进行了EDX 元素分析, 选择 支撑体和膜层独有的 $\mathrm{Ba}$ 和 $\mathrm{Co}$ 元素作为分析对象, 结 果如图4(b), 支撑体和膜层界面有稍微元素扩散, 这 是因为高温烧结过程中膜层与支撑体之间发生一定 的固相反应。由于元素扩散不严重, 不会影响到的膜 性能, 另外界面发生一定程度的固相反应可以提高膜 层与支撑体之间的结合力。从膜的外表面照片(图4(c)) 上可以看到陶瓷膜形成明显的晶界, 膜表面致密无 缺陷。在经历同样烧结制度的SBFM中空纤维支撑体 仍然还是多孔结构, 表面分布着大量微小气孔如图 4(d)。通过气密性检测发现担载型SCFNb/SBFM中空 纤维氧渗透膜的气密性良好, 而同样烧结的SBFM中 空纤维支撑体却具有透气效果。
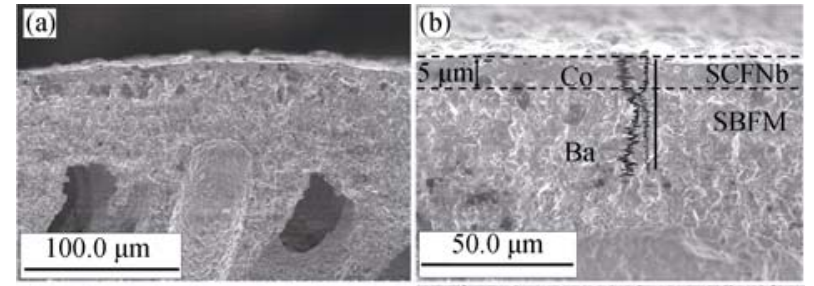

(c)
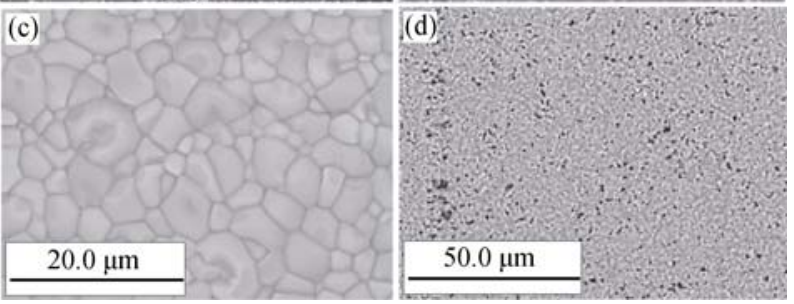

图 4 担载型 $\mathrm{SCFNb} / \mathrm{SBFM}$ 中空纤维氧渗透膜和支撑体的 SEM 照片

Fig. 4 SEM images of SCFNb/SBFM supported hollow fiber membrane

(a) Cross section; (b) EDX analysis of cross section; (c) Outside surface of membrane; (d) Hollow fiber support

\section{4 担载型中空纤维膜的氧渗透性能分析}

实验对担载型 $\mathrm{SCFNb} / \mathrm{SBFM}$ 中空纤维氧渗透 膜氧渗透性能进行了考察, 图 5 给出了担载型 $\mathrm{SCFNb} / \mathrm{SBFM}$ 中空纤维氧渗透膜的氧渗透通量随 着温度变化的关系曲线, 测试温度为 $800 \sim 900^{\circ} \mathrm{C}$, 氧分压梯度为 $0.21 \times 10^{5} / 1 \times 10^{2} \mathrm{~Pa}$ 。如图 5 所示, 膜 管的氧渗透通量随着温度升高显著增加, 氧渗透通 量的增加主要归功于膜表面氧交换速率和透氧膜体 相氧扩散速率的增加。在 $800^{\circ} \mathrm{C}$ 时, 非对称膜的氧通 量达到 $0.34 \mathrm{~mL} /\left(\mathrm{cm}^{2} \cdot \mathrm{min}\right)$, 是 $\mathrm{Wu}$ 等 ${ }^{[8]}$ 报道的 $\mathrm{SCFNb}$ 中空纤维膜氧通量的 2 倍。根据 Arrhenius 曲线, 计 算 $800 \sim 900^{\circ} \mathrm{C}$ 温度范围内担载型 $\mathrm{SCFNb} / \mathrm{SBFM}$ 中空 纤维氧渗透膜的氧渗透活化能为 $91.9 \mathrm{~kJ} / \mathrm{mol}$ 。

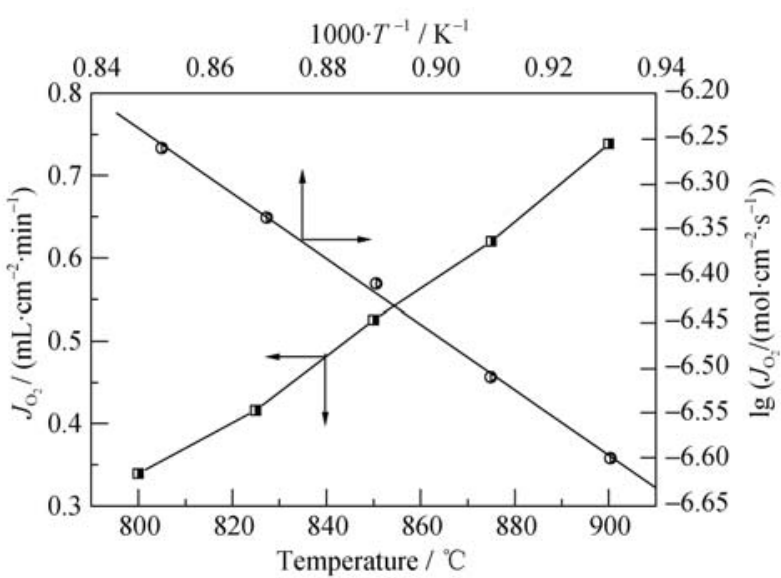

图 5 担载型 $\mathrm{SCFNb} / \mathrm{SBFM}$ 中空纤维氧渗透膜的氧渗透性能 Fig. 5 Temperature dependence of oxygen fluxes through $\mathrm{SCFNb}$ /SBFM supported hollow fiber 


\section{5 担载型中空纤维膜反应器的甲烷部分氧 化性能评价}

实验对担载型 $\mathrm{SCFNb} / \mathrm{SBFM}$ 中空纤维氧渗透膜 的甲烷部分氧化反应性能也进行了考察, 测试温度 范围为 750 $900^{\circ} \mathrm{C}$ 。在每个温度点停留 $1 \mathrm{~h}$ 以确保结 果的准确性。图 6 给出了反应温度对 $\mathrm{CH}_{4}$ 转化率、 $\mathrm{CO}$ 选择性以及氧渗透通量的影响。如图 6 所示, $\mathrm{CH}_{4}$ 转化率和氧渗透通量随着温度升高而增大。随着温 度升高, 体相扩散和表面交换速度增加从而导致氧 渗透通量的增加。氧渗透通量的增加有助于 $\mathrm{CH}_{4}$ 转 化率的提高, 因而氧渗透通量的增加自然会引起 $\mathrm{CH}_{4}$ 转化率的增大。而 $\mathrm{CO}$ 的选择性随着温度升高而 略微降低。这是由于温度升高导致氧渗透通量增大, 进料中甲烷浓度保持恒定使得高温下 $\mathrm{CH}_{4} / \mathrm{O}_{2}$ 比的降 低, 进而导致 $\mathrm{CO}$ 被过度氧化为 $\mathrm{CO}_{2}$ 。 $850^{\circ} \mathrm{C}$ 条件下, $\mathrm{CH}_{4}$ 转化率为 $81 \%, \mathrm{CO}$ 选择性为 $95.33 \%$, 氧渗透通 量为 $4.83 \mathrm{~mL} /\left(\mathrm{cm}^{2} \cdot \mathrm{min}\right)$, 产品的 $\mathrm{H}_{2} / \mathrm{CO}$ 达到了 1.38 。

\section{6 中空纤维膜反应器的长期稳定性分析}

膜的长期稳定性是考察性能的一个非常重要 的方面, 尤其是在工业应用过程中膜的稳定性直 接关系到其能否实现工业化。选定在 $850^{\circ} \mathrm{C}, \mathrm{CH}_{4}$ 、 $\mathrm{He}$ 和空气流量分别为 $4 、 18$ 和 $150 \mathrm{~mL} / \mathrm{min}$ 条件下 进行膜的长期稳定性测试。如图 7 所示, 经过大约 $2 \mathrm{~h}, \mathrm{CH}_{4}$ 转化率、 $\mathrm{CO}$ 选择性和氧渗透通量达到稳 态, 分别约为 $81 \% 、 93 \%$ 和 $4.5 \mathrm{~mL} /\left(\mathrm{cm}^{2} \cdot \mathrm{min}\right)$, 且在 $200 \mathrm{~h}$ 的测试过程中几乎保持不变, 之后手动停止 反应, 温度降到室温后将膜组件取出, 发现膜管 仍然完好, 没有破损, 这说明担载型 $\mathrm{SCFNb}$ / SBFM 中空纤维氧渗透膜的设计提高了膜在甲烷 部分氧化过程中的稳定性, 与课题组前期研究

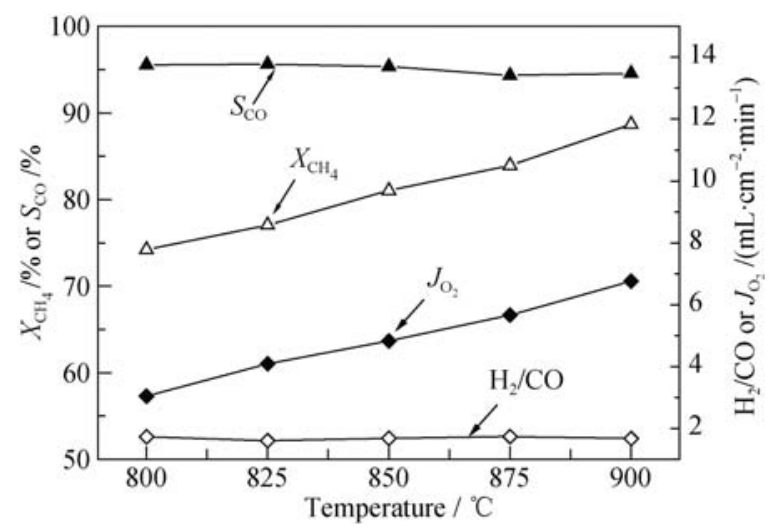

图 6 担载型中空纤维膜的反应性能与温度关系

Fig. 6 CO selectivity, $\mathrm{CH}_{4}$ conversion, $\mathrm{H}_{2} / \mathrm{CO}$ and $\mathrm{O}_{2}$ permeation flux as a function of temperature

Reaction side: $\mathrm{He}=20 \mathrm{~mL} / \mathrm{min}, \mathrm{CH}_{4}=3 \mathrm{~mL} / \mathrm{min}$; Air side: air $=150 \mathrm{~mL} / \mathrm{min}$

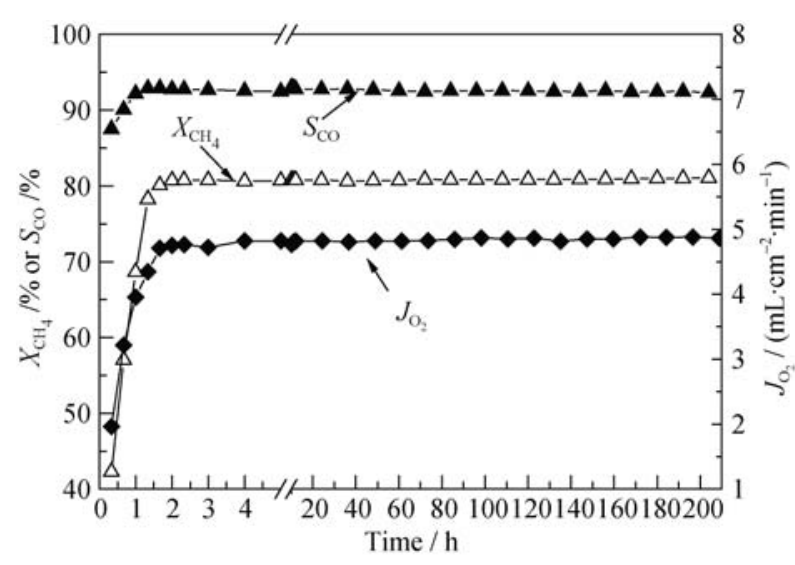

图 7 担载型 $\mathrm{SCFNb} / \mathrm{SBFM}$ 中空纤维氧渗透膜稳定性考察

Fig. 7 Long-term performance of reaction in the $\mathrm{SCFNb} /$ SBFM supported hollow fiber membrane reactor at $850^{\circ} \mathrm{C}$

工作 ${ }^{[16]}$ 的实验结果一致, SBFM 支撑体能有效地增 强 SCFNb 膜层在还原性气氛下的稳定性。一方面 是由于耐还原的 SBFM 多孔支撑体与 $\mathrm{SCFNb}$ 致密 膜层紧密结合避免了还原性气氛对 SCFNb 膜层的 直接冲击, 并且 SBFM 也是一个良好的离子导体, 部分氧离子能通过 BSFM 传递到多孔层, 减少了 还原性气体与致密层膜表面的接触; 另一方面是 由于 $\mathrm{SCFNb}$ 具有非常好的氧渗透性能, 确保了在 其表面始终存在一个非零氧分压，阻碍了还原性 气体对表面的腐蚀 ${ }^{[15]}$ 。

\section{3 结论}

以单一海绵孔/指状孔结构的 SBFM 中空纤维为 支撑体，采用旋转喷涂结合共烧结技术，制备出膜 层致密无缺陷且膜厚 $5 \mu \mathrm{m}$ 的担载型中空纤维氧渗透 膜。研究显示, 担载型 $\mathrm{SCFNb} / \mathrm{SBFM}$ 中空纤维氧渗 透膜氧通量是 $\mathrm{SCFNb}$ 中空纤维膜的 2 倍, $900^{\circ} \mathrm{C}$ 下氧 渗透通量达到 $0.74 \mathrm{~mL} /\left(\mathrm{cm}^{2} \cdot \mathrm{min}\right)$ 。在 $850^{\circ} \mathrm{C}$ 时, 膜的 甲烷部分氧化反应稳定操作超过 $200 \mathrm{~h}$, 研究表明, 担载型 SCFNb/SBFM 中空纤维氧渗透膜具有较高的 氧通量及良好的甲烷部分氧化膜反应稳定性。

\section{参考文献:}

[1] BOUWMEESTER H J M, BURGGRAAF A J. Fundamentals of Inorganic Membrane Science Technology. Elsevier, Amsterdam, 1996: 435-515.

[2] JIN W Q, LI S G, HUANG P, et al. Preparation of an asymmetric perovskite-type membrane and its oxygen permeability. Journal of Membrane Science, 2001, 185(2): 237-243. 
[3] ITO W, NAGAI T, SAKON T. Oxygen separation from compressed air using a mixed conducting perovskite-type oxide membrane. Solid State Ionics, 2007, 178(11/12): 809-816.

[4] KAWAHARA A, TAKAHASHI Y, HIRANO Y, et al. High oxygen permeation rate in $\mathrm{La}_{0.6} \mathrm{Sr}_{0.4} \mathrm{Ti}_{0.3} \mathrm{Fe}_{0.7} \mathrm{O}_{3-\delta}$ thin membrane on a porous support with multichannel structure for $\mathrm{CH}_{4}$ partial oxidation. Industrial \& Engineering Chemistry Research, 2010, 49(12): $5511-5516$

[5] FAN CHUAN-GANG, HUANG XIANG-XIAN, LIU WEI, et al. Preparation and oxygen permeation for $\mathrm{SrCo}_{0.8} \mathrm{Fe}_{0.2} \mathrm{O}_{3-\delta}$ tubular asymmetric membrane. Journal of Inorganic Materials, 2008, 23(6): $1221-1224$

[6] ZYDORCZAK B, WU Z T, LI K. Fabrication of ultrathin $\mathrm{La}_{0.6} \mathrm{Sr}_{0.4} \mathrm{Co}_{0.2} \mathrm{Fe}_{0.8} \mathrm{O}_{3-\delta}$ hollow fibre membranes for oxygen permeation. Chemical Engineering Science, 2009, 64(21): 4383-4388.

[7] WANG B, ZYDORCZAK B, POULIDI D, et al. A further investigation of the kinetic demixing/decomposition of $\mathrm{La}_{0.6} \mathrm{Sr}_{0.4} \mathrm{Co}_{0.2} \mathrm{Fe}_{0.8} \mathrm{O}_{3-\delta}$ oxygen separation membranes. Journal of Membrane Science, 2011, 369(1/2): 526-535.

[8] WU Z T, OTHMAN N H, ZHANG G R, et al. Effects of fabrication processes on oxygen permeation of $\mathrm{Nb}_{2} \mathrm{O}_{5}$-doped $\mathrm{SrCo}_{0.8} \mathrm{Fe}_{0.2} \mathrm{O}_{3-\delta}$ micro-tubular membranes. Journal of Membrane Science, 2013, 442(1): $1-7$.

[9] LIANG F Y, JIANG H Q, SCHIESTEL T, et al. High-purity oxygen production from air using perovskite hollow fiber membranes. Industrial \& Engineering Chemistry Research, 2010, 49(19): 9377-9384.

[10] CHEN XIN-ZHI, YU LING-HUI, LIU QIAO-SHENG, et al. Preparation and application of perovskite hollow fiber oxygen permeable membrane. Journal of Inorganic Materials, 2008, 23(6): 1216-1220.

[11] LIAO Q, ZHENG Q, XUE J, et al. U-Shaped $\mathrm{BaCo}_{0.7} \mathrm{Fe}_{0.2} \mathrm{Ta}_{0.1} \mathrm{O}_{3-\delta}$ hollow-fiber membranes with high permeation for oxygen separa- tion. Industrial \& Engineering Chemistry Research, 2012, 51(46): $15217-15223$.

[12] TAN XIAO-YAO, MENG BO, YANG NAI-TAO. Preparation and characteristics of ceramic hollow fiber membranes for oxygen permeation. Journal of Inorganic Materials, 2006, 21(1): 245-249.

[13] LIU N, TAN X Y, MENG B, et al. Honeycomb-structured perovskite hollow fibre membranes with ultra-thin densified layer for oxygen separation. Separation and Purification Technology, 2011, 80(2): 396-401.

[14] PAN H P, LI L P, DENG X, et al. Improvement of oxygen permeation in perovskite hollow fibre membranes by the enhanced surface exchange kinetics. Journal of Membrane Science, 2013, 428(1): $198-204$.

[15] WANG H H, FELDHOFF A, CARO J, et al. Oxygen selective ceramic hollow fiber membranes for partial oxidation of methane. AIChE Journal, 2009, 55(10): 2657-2664.

[16] JIANG W, ZHANG G R, LIU Z K, et al. A novel porous-dense dual-layer composite membrane reactor with long-term stability. AIChE Journal, 2013, 59(11): 4355-4363.

[17] ZHANG G R, LIU Z K, ZHU N, et al. A novel $\mathrm{Nb}_{2} \mathrm{O}_{5}$-doped $\mathrm{SrCo}_{0.8} \mathrm{Fe}_{0.2} \mathrm{O}_{3-\delta}$ oxide with high permeability and stability for oxygen separation. Journal of Membrane Science, 2012, 405(1): 300-309.

[18] LIU Z K, ZHANG G R, DONG X L, et al. Fabrication of asymmetric tubular mixed-conducting dense membranes by a combined spin-spraying and co-sintering process. Journal of Membrane Science, 2012, 415(1): 313-319.

[19] QI LV, DONG XUE-LIANG, LIU ZHENG-KUN, et al. Preparation and oxygen permeation of $\mathrm{SrCo}_{0.4} \mathrm{Fe}_{0.4} \mathrm{Zr}_{0.2} \mathrm{O}_{3-\delta}$ mixed-conducting hollow fibre membrane. Journal of Inorganic Materials, 2010, 25(8): $871-876$

[20] LIN Y S, BURGGRAAF A J. Experimental studies on pore size change of porous ceramic membranes after modification. Journal of Membrane Science, 1993, 79(1): 65-82. 